\title{
Steel for gas and oil pipelines resistant to destruction in hydrogen sulphide-containing media
}

\author{
Yury Matrosov ${ }^{1}$, Ivan Shabalov ${ }^{1,2}$, Alexey Kholodnyi ${ }^{1, *}$, and Valery Velikodnev ${ }^{3}$ \\ ${ }^{1}$ I.P. Bardin Central Research Institute for Ferrous Metallurgy, 105005 Moscow, Russia \\ ${ }^{2}$ Association of Pipe Manufacturers, 115088 Moscow, Russia \\ ${ }^{3}$ Center for Examination of Pipeline Systems and Engineering, 127051 Moscow, Russia
}

\begin{abstract}
About a third reserves of the natural gas and oil contain $\mathrm{H}_{2} \mathrm{~S}$ impurities, which, in the presence of moisture, form an acidic medium and can lead to pipeline destruction by the mechanisms of hydrogen-induced cracking (HIC) and sulfide stress cracking (SSC). With the growing number of hydrocarbon fields being developed with a high content of $\mathrm{H}_{2} \mathrm{~S}$, the demand for gas and oil large diameter pipes grade from BMS to X70MS with high resistance to HIC and SSC increases. Comprehensive studies have been carried out in laboratory and industrial conditions to determine the effect of the chemical composition and thermomechanical processing on the microstructure, mechanical properties and resistance to HIC of rolled plates from low-alloy pipe steels. Optimal concentration of segregating elements $(\mathrm{C} \leq 0.06 \%, \mathrm{Mn} \leq 1.00 \%)$, and schedules of accelerated cooling after controlled rolling $\left(\mathrm{T}_{\mathrm{sc}} \geq \mathrm{Ar}_{3} ; \mathrm{T}_{\mathrm{fc}}=520 \pm 30^{\circ} \mathrm{C} ; \mathrm{V}_{\mathrm{c}} \geq 20^{\circ} \mathrm{C} / \mathrm{c}\right)$ provide the high resistance to destruction in $\mathrm{H}_{2} \mathrm{~S}-$ containing media due the formation of a homogeneous microstructure without developed central segregation heterogeneity was determined. According to the research results, the technology for manufacturing of plates for largediameter pipes grade X52MS, X56MS, X60MS, and X65MS ordered for sour service was developed.
\end{abstract}

\section{Introduction}

In recent years, the number of developed gas and oil fields with an increased content of hydrogen sulfide grows, while previously such fields were considered to be of little use for operation. This is explained by the depletion of "clean" fields, which have been the main source of hydrocarbons for many decades. At the same time, there is a growing demand for gas-oil pipes, which are much more cracking resistant in an aggressive hydrogen sulfide-containing medium, the so-called "sour gas", than the steels used to manufacture pipes that transport non-aggressive natural gas or oil. To prevent damage of pipelines transmitting "sour gas" from fields to plants for its purification from harmful $\mathrm{H}_{2} \mathrm{~S}, \mathrm{CO}_{2}$, etc. impurities, special requirements have been developed for the resistance of pipe metal to failure in $\mathrm{H}_{2} \mathrm{~S}$-containing media. The fulfilment of such requirements is necessary to ensure a long-life operation of the pipes.

\section{Effect of $\mathrm{H}_{2} \mathrm{~S}$-containing media on pipe steels}

Upon contact with moisture, hydrogen sulfide contained in gas and oil dissolves in it and dissociates $\left(\mathrm{H}_{2} \mathrm{~S} \rightarrow \mathrm{HS}^{-}\right.$ $+\mathrm{H}^{+}$) to form $\mathrm{H}^{+}$ions, thus reducing the $\mathrm{pH}$ and forming an acidic corrosive medium. The acidic medium enters an electrochemical reaction with the steel and causes an anodic reaction such as ionization of iron $\left(\mathrm{Fe} \rightarrow \mathrm{Fe}^{2+}+\right.$ $\left.2 \mathrm{e}^{-}\right)$. The reduced hydrogen atoms $\left(\mathrm{H}^{+}+\mathrm{e}^{-} \rightarrow \mathrm{H}\right)$ are attracted to the metal surface and adsorbed by it due to the presence of free electrons. Upon the contact with the metal surface, the atomic hydrogen can pass to the $\mathrm{H}_{2}$ molecular form or can be absorbed by (penetrate into) the metal. The hydrogen atoms, which have diffused into the metal volume, are distributed in a certain way among the metal atoms. Hydrogen atoms in metal act as interstitial impurity. They are present in the crystal lattice between iron atoms and alloying elements or are accumulated in "traps" such as interfaces between nonmetallic inclusions and matrix and other defects in the metal structure (grain boundaries and interphases, pores, etc.). Atomic hydrogen can pass into the molecular form of $\mathrm{H}_{2}$ in the regions of the heterogeneity of the steel matrix. Since the resulting hydrogen gas occupies a substantial volume, the process of hydrogen transition from the atomic state to the molecular one leads to the excessive internal pressure, to high internal tensile stresses and, in critical cases, to the formation of microcracks. After the nucleation stage, the hydrogen initiated crack propagates by the mechanism of the coalescence of several pores. The resulting cracks propagate mainly through hard and brittle microstructure constituents [1-4].

Hydrogen in the atomic and/or molecular state in steel can lead to the fracture of steel mainly by two mechanisms: cracking occurring without stress hydrogen-induced cracking (HIC); cracking occurring upon the action of residual and/or applied stress - sulfide stress cracking (SSC). HIC is the lamellar plate cracking

\footnotetext{
* Corresponding author: kholodnyi.aa $@$ gmail.com
} 
caused by the diffusion of atomic hydrogen, its accumulation in traps, and the formation of molecular hydrogen. Cracking occurs due to a sharp increase in pressure as a result of the recombination of hydrogen from the atomic to the molecular state. Such cracks appear in the regions with a high density of non-metallic inclusions, especially of flat shape, and the areas of anomalous structure (for example, banded structure) resulting from the segregation of impurities and alloying elements in steel. SSC is the cracking of metal under the action of tensile stresses in the sour medium. Sulfide stress cracking is associated with the embrittlement of the metal by atomic hydrogen. Atomic hydrogen decreases the plasticity of steel and increases its susceptibility to cracking. In the case of steel failure by the SSC mechanism, the crack formation caused by the pressure of molecular hydrogen should also be taken into account. Such failure is typical of high-strength metal materials and the zones of increased hardness of weld.

\section{Factors affecting the cracking resistance of pipe steels in $\mathrm{H}_{2} \mathrm{~S}$ - containing media}

The negative effect of aggressive $\mathrm{H}_{2} \mathrm{~S}$-containing wet media in transported gas and oil causes disruption of continuity and failure of pipes by the mechanisms of hydrogen-induced cracking (HIC), sulfide stress cracking (SSC) and is determined by a number of factors are related to the metallurgical properties of the pipe metal and include the chemical composition, microstructure, the shape and distribution of nonmetallic inclusions, strength properties, internal stresses.

The accumulation of atomic hydrogen, which saturates the steel, as well as its recombination into the molecular form occur at structure discontinuities such as interphase interfaces between non-metallic inclusions and steel matrix. This leads to the appearance of internal gas pressure and tensile stresses, which, after exceeding a certain critical level, initiate the crack nucleation. The stress level depends on the shape of the inclusions, their size, quantity, distribution, and distance from the plate/pipe surface. Due to the specific features of crystallization and solidification of a continuously cast slab, most non-metallic inclusions, harmful impurities $(\mathrm{S}, \mathrm{P})$, and alloying elements $(\mathrm{Mn}, \mathrm{Nb}, \mathrm{C})$ are concentrated in the axial zone. Predominantly, this leads to hydrogen-induced cracking in the axial segregation region. Therefore, special attention upon the production of continuously cast slabs for plates intended for sour gas resistant pipes is paid to the measures aimed at increasing steel cleanliness from non-metallic inclusions and reducing centreline segregation heterogeneity.

The main non-metallic inclusions detrimentally affecting the cracking resistance of the plates from pipe steels in $\mathrm{H}_{2} \mathrm{~S}$-containing media are as follows: inclusions based on manganese sulfide $(\mathrm{MnS})$; inclusions of a complex composition based on niobium and titanium carbonitrides $(\mathrm{Ti}, \mathrm{Nb})(\mathrm{C}, \mathrm{N})$; oxide-sulfide inclusions of complex composition. The modern level of metallurgical technology of steelmaking, out-of-furnace processing, and continuous casting can provide minimum contamination of the slab by non-metallic inclusions.

High cleanliness of the steel is an essential, but not final condition of the high HIC resistance of plates. The other important factor is the type of microstructure formed in the steel at the stage of thermomechanical processing. According to the results of the study, it was concluded that the predominant sites of the nucleation and propagation of hydrogen cracks in the plates of high cleanliness from non-metallic inclusions are extended segregation bands of increased hardness in the zone of central structural heterogeneity of the plates. In the plates produced by different thermomechanical processing regimes, the hydrogen-induced cracks propagate in the zone of central structural heterogeneity along coarse extended segregation bands with regions of high-carbon structures such as lamellar pearlite, degenerate pearlite (fig. 1), high-carbon upper bainite, twinned high-carbon martensite with retained austenite, and acicular bainitic ferrite with interlayers of retained austenite along the lath boundaries [1-4].

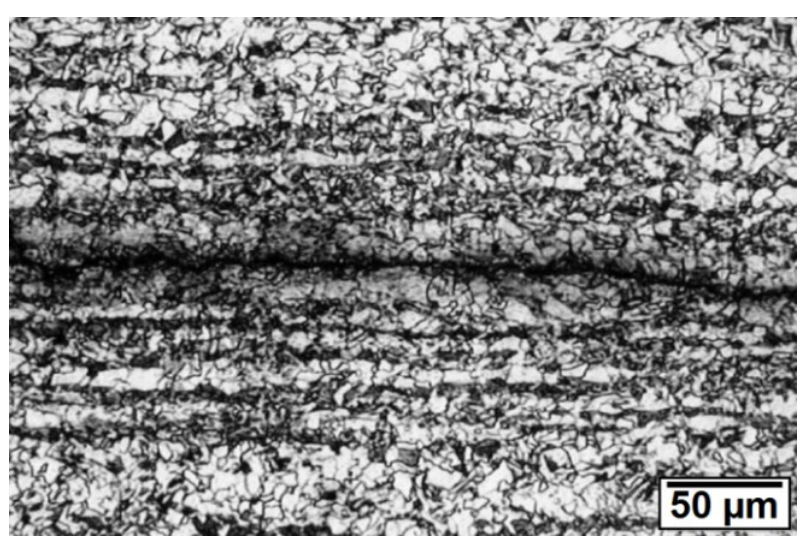

Fig. 1. Hydrogen-induced crack in the centerline segregation zone of the plate

Microstructure of the base metal free from structural heterogeneity in the form of banding does not show a tendency to hydrogen cracking. If the plate metal is manufactured with the completion of deformation in a finishing stand in the two-phase $(\gamma+\alpha)$ region, the formation of banded structure can occur not only in the central segregation zone, but also in the base metal. Such elongated bands in the base metal can be also the sites of the nucleation and propagation of hydrogen cracks.

\section{Effect of chemical composition on the $\mathrm{HIC}$ resistance of rolled plates}

The chemical composition of steel, especially the content of strongly segregating elements - C and $\mathrm{Mn}$ - affects the degree of central segregation in the slab and, as a consequence, the segregation heterogeneity in the axial zone of the plates. On the other hand, the chemical composition both in the base metal and in the axial zone affects the kinetics of the polymorphic transformation upon cooling of the plate after rolling, and this also determines the degree of chemical and microstructural heterogeneity of the rolled products $[4,5]$. 


\subsection{Carbon content}

Carbon is related to the elements that most strongly segregate in the axial slab zone upon crystallization during casting. Simultaneously with an increase in its content in steel, the susceptibility to primary segregation of elements such as $\mathrm{Mn}, \mathrm{S}, \mathrm{P}$, and $\mathrm{Nb}$ increases. Therefore, the carbon concentration is an important factor affecting the degree of central segregation heterogeneity and the HIC resistance of the plates from low-carbon low-alloy pipe steels.

The effect of carbon content changing from 0.04 to $0.08 \%$ at comparable manganese contents in a range of $1.25-1.35 \%$ on the central segregation heterogeneity and HIC resistance of the plates $22 \mathrm{~mm}$ thick manufactured by controlled rolling followed by accelerated cooling has been studied. The plates were cooled from the austenite field to the lower temperatures of bainitic transformation range at a rate of more than $20^{\circ} \mathrm{C} / \mathrm{s}$.

The base metal of the plates with a carbon content of 0.04 and $0.08 \%$ had a homogeneous ferritic-bainitic microstructure. The carbon content substantially affects the microstructure of the axial zone of the plates. At $0.04 \% \mathrm{C}$, a slight structural heterogeneity is seen in the axial zone in the form of a narrow segregation band. As the carbon content increases to $0.08 \%$, the central structural heterogeneity becomes more pronounced, which is expressed in an increase in the number of segregation bands.

A decrease in the carbon content, other things being equal, resulted in a substantial increase in the HIC resistance of the plates. The crack length ratio (CLR) parameter on average decreased from $12.5 \%$ at $0.08 \% \mathrm{C}$ to zero at $0.04 \% \mathrm{C}$ (fig. 2 ).

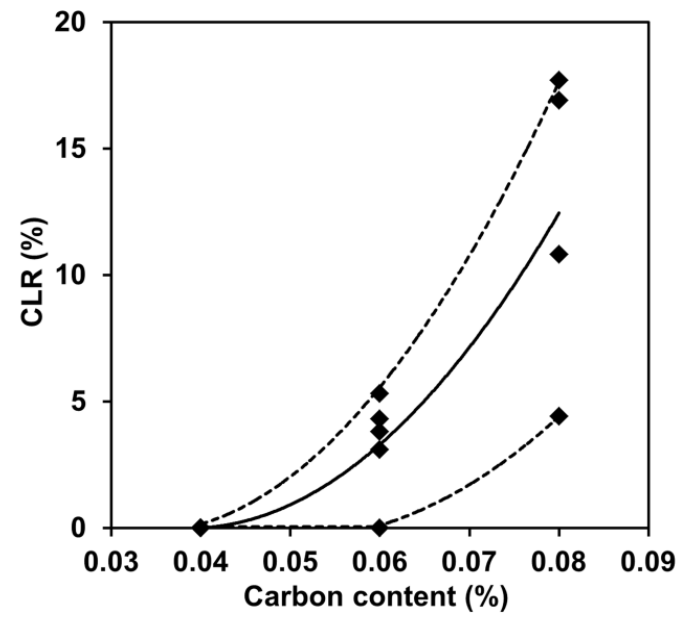

Fig. 2. Effect of carbon content on the crack length ratio

\subsection{Manganese content}

Manganese is the main alloying element in low-alloy pipe steels. Depending on the pipe application and grade, its content is usually in a range of $1.40-1.90 \%$. However, for steels resistant to cracking in $\mathrm{H}_{2} \mathrm{~S}$-containing media, its content is maintained at a lower level (usually no higher than $1.30 \%$ ), which is caused by its increased tendency to segregation upon solidification of liquid metal during continuous casting.

The effect of manganese concentration on the the HIC susceptibility of plates was studied on the steels contained from 0.65 to $1.35 \%$ manganese at $0.06 \%$ carbon. Plates $15-25 \mathrm{~mm}$ thick were divided into two groups in accordance with the technological regimes of accelerated cooling after controlled rolling:

- regime No. 1: $\mathrm{T}_{\mathrm{sc}} \geq \mathrm{Ar}_{3}, \mathrm{~T}_{\mathrm{fc}}=540-640^{\circ} \mathrm{C}, \mathrm{V}_{\mathrm{c}}=12$ $18^{\circ} \mathrm{C} / \mathrm{s}$ (fig. $3 \mathrm{a}$ );

- regime No. 2: $\mathrm{T}_{\mathrm{sc}} \geq \mathrm{Ar}_{3}, \mathrm{~T}_{\mathrm{fc}}=490-530^{\circ} \mathrm{C}, \mathrm{V}_{\mathrm{c}}=22$ $26^{\circ} \mathrm{C} / \mathrm{s}$ (fig. $3 \mathrm{~b}$ ).
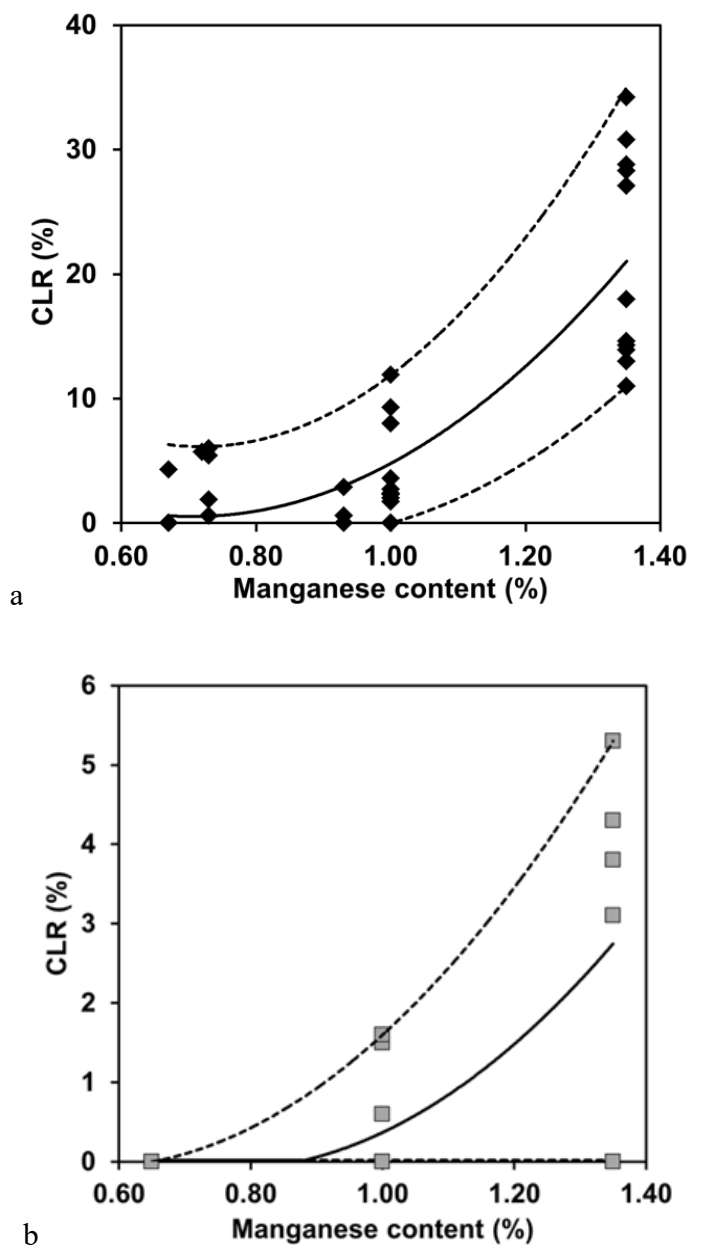

Fig. 3. Effect of manganese content on the crack length ratio: a - regime No. $1\left(\mathrm{~T}_{\mathrm{sc}} \geq \mathrm{Ar}_{3}, \mathrm{~T}_{\mathrm{fc}}=540-640^{\circ} \mathrm{C}, \mathrm{V}_{\mathrm{c}}=12-18^{\circ} \mathrm{C} / \mathrm{s}\right)$ $\mathrm{b}$ - regime No. $2\left(\mathrm{~T}_{\mathrm{sc}} \geq \mathrm{Ar}_{3}, \mathrm{~T}_{\mathrm{fc}}=490-530^{\circ} \mathrm{C}, \mathrm{V}_{\mathrm{c}}=22-26^{\circ} \mathrm{C} / \mathrm{s}\right)$

The plates were rapidly cooled from the single-phase $\gamma$ field at both treatment regimes, but regime No. 1 used a higher finish temperature of accelerated cooling and a lower cooling rate. The estimation of the effect of manganese on the susceptibility to HIC of the plates processed by the above regimes shows that, in both cases, the reduction in manganese content positively affects the HIC resistance of the plates from the test steels. At the same time, a decrease in the finish temperature of the accelerated cooling and an increase in cooling rate (plates processed by regime No. 2) substantially increase the HIC resistance compared to 
that of the plates processed by regime No. 1. For example, at a manganese content of $1.00 \%$, the treatment regime No. 1 provided an average CLR of $5 \%$ (maximum 12\%), while after the treatment by regime No. 2, CLR was $0.5 \%$ (maximum $1.6 \%$ ). On average, the HIC parameters of the plates treated by regime No. 2 were smaller by a factor of about eight than those of the plates treated by regime No. 1 in the entire range of manganese contents used.

After treatment by regime No. 2, the HIC parameter reached zero at a manganese content of about $0.90 \%$. Thus, the additive effect of a decrease in manganese content and a simultaneous decrease in the finish temperature of the accelerated cooling and an increase in the cooling rate has been established for an increase in the HIC resistance of the plates.

\section{Effect of post-deformation cooling on the HIC resistance of rolled plates}

For the development of the thermomechanical treatment regimes for HIC resistant plates from pipe steels, their effect on the formation of the base metal microstructure responsible for the mechanical properties of the steel and on the axial zone microstructure responsible for the HIC resistance of the plates are taken into account. The structure formation in the central segregation zone and in the base metal of the plates is mainly affected by the conditions of hot plastic deformation and cooling. The microstructure formation in the centerline zone is characterized by some specific features associated, first of all, with a higher content of segregating elements, which is inherited from continuously cast slabs. The effect of the post-deformation cooling parameters such as start temperature of accelerated cooling $\left(\mathrm{T}_{\mathrm{sc}}\right)$, finish temperature of accelerated cooling $\left(\mathrm{T}_{\mathrm{fc}}\right)$, and cooling rate $\left(\mathrm{V}_{\mathrm{c}}\right)$ on the HIC resistance of rolled plates was investigated $[4,6]$.

\subsection{Start temperature of accelerated cooling}

If the start temperature of accelerated cooling belongs to the $\gamma$ field, the HIC parameters tend to zero (fig. 4). On the contrary, as $\mathrm{T}_{\mathrm{sc}}$ decreases to the $(\gamma+\alpha)$ field, the HIC parameters increase. The detected hydrogen-induced cracks are present in the axial rolling zone. The crack propagation occurs along the interphase interfaces between ferrite and hard high-carbon structures.

On the basis of the experimental results one conclude that, as the start temperature of accelerated cooling is above the $\mathrm{Ar}_{3}$ temperature, the microstructure is more uniform over the plate thickness and less susceptible to HIC than that formed upon accelerated cooling from the temperature below $\mathrm{Ar}_{3}$. This is explained by the enrichment of undecomposed austenite with carbon and alloying elements upon the $\gamma \rightarrow \alpha$ polymorphic transformation occurring upon slow cooling. The decomposition of such austenite can occur in the pearlitic and bainitic regions, as well as with the formation of the MA constituent. In this case, the difference between the steel matrix and the segregation bands in hardness increases, which promotes the nucleation and propagation of hydrogen-induced cracks.

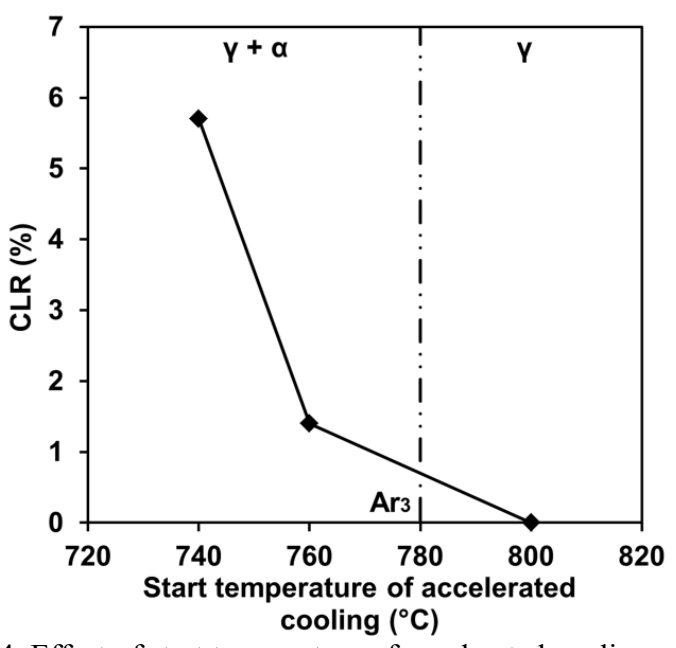

Fig. 4. Effect of start temperature of accelerated cooling on the crack length ratio figures.

\subsection{Finish temperature of accelerated cooling}

The results of the study show that, in the case of relatively high finish temperatures of accelerated cooling, the structure formed in the axial zone of the plates contains a second phase in the form of coarse pearlite bands. Accelerated cooling to temperatures belonging to the lower range of bainitic transformation leads to the formation of bands consisting of high-carbon bainite. At a lower finish temperature of accelerated cooling, bands with regions of the MA constituent can form. Such high-carbon structures are favourable for the nucleation and propagation of hydrogen-induced cracks. The optimum range of the finish temperatures of accelerated cooling for the steels under study is the lower range of the bainitic transformation region (in this case, $\mathrm{T}_{\mathrm{fc}} \sim 500^{\circ} \mathrm{C}$ ). The ferritic-bainitic microstructure formed in the axial zone at such temperatures is more uniform and less susceptible to HIC than the structures formed upon cooling to higher or lower temperatures (fig. 5).

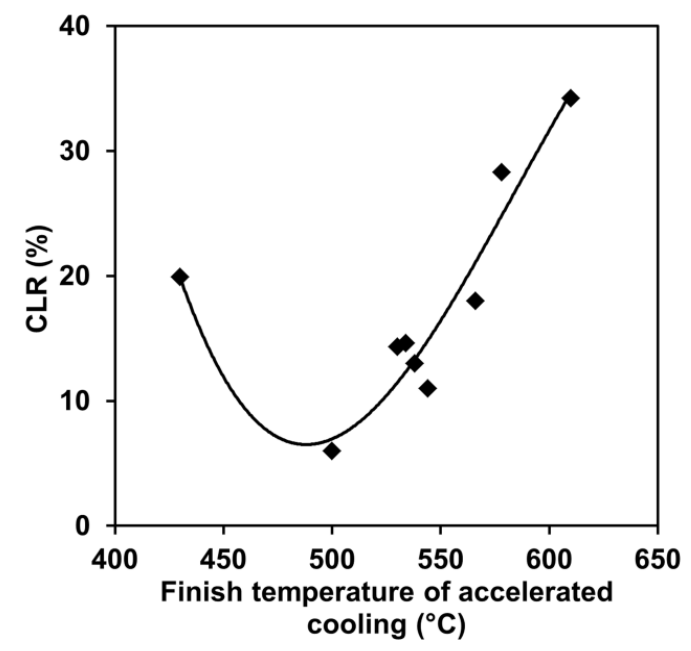

Fig. 5. Effect of finish temperature of accelerated cooling on the crack length ratio 


\subsection{Cooling rate}

With an increase in the cooling rate from 2 to $25^{\circ} \mathrm{C} / \mathrm{s}$, a significant increase in the HIC resistance of the plates (fig. 6). An increase in the microstructural homogeneity over the plate thickness and an increase in the HIC resistance of the plates with increasing cooling rate is associated with a decrease in the rate of the diffusion of carbon and other elements into austenite upon phase transformations. This allows one to reduce the amount of non-decomposed austenite regions enriched with alloying elements, primarily with carbon, and to reduce the number and extension of the bands consisting of unfavourable transformation products such as pearlite, high-carbon bainite, and MA-constituent, which adversely affect the HIC resistance.

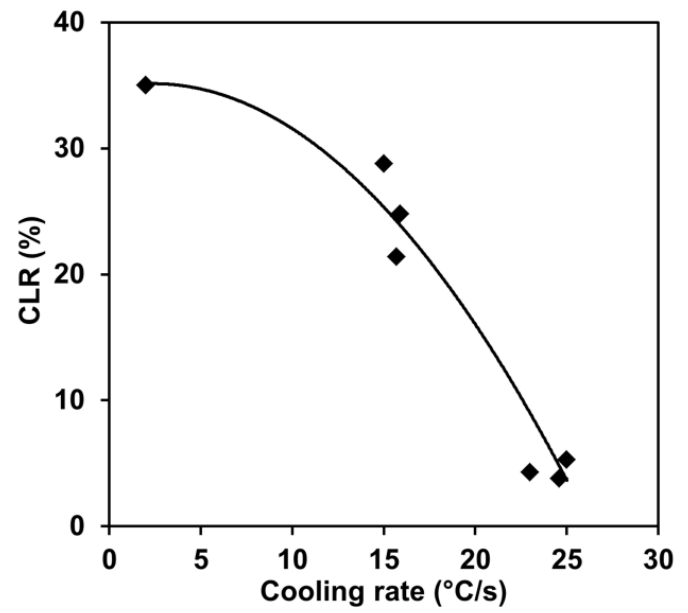

Fig. 6. Effect of cooling rate on the crack length ratio

\section{Integrated production concept of pipe steels for sour service}

As the requirements for sour service gas pipeline steels become more severe, all steps in the manufacture of such steels have to be optimized, especially with the aim of a clean steel with minimized inclusions and with homogeneity of microstructure which is a consequence of reduced center segregation of slabs and of adapted manufacturing technology. In order to provide consistent HIC resistance the production of sour service steels must relies on an integrated overall production concept. This involves the steel- and plate-making as well as the application of a specific quality assurance system.

The general strategy of production for sour service steels relies on: vacuum treatment by tank degassing; very low sulfur content $(\leq 0.0001 \%)$ and $\mathrm{CaSi}$ treatment for shape control to prevent $\mathrm{MnS}$; minimization of segregation and contamination by inclusions during continuous casting of slabs; controlled rolling with accelerated cooling after final rolling with specific temperatures; microstructure control by limit of $\mathrm{C} \leq$ $0.05 \%, \mathrm{Mn} \leq 1.30 \%$ and $\mathrm{P} \leq 0.015 \%$ contents.

During production certain deviations or incidents are possible having significant influence on HIC properties and no or minor influence on other properties. For that reason a special adapted quality control system for HIC resistant steel must be installed that is able to state a deviation of actual values and to decide if such plates must be prohibited from release and delivery or additionally tested to prove conformity with specification. With all the measure explained above it is possible to produce HIC resistant steel plates with consistent property profiles even in big quantities [7].

\section{Manufacturing technology of steels for pipes ordered for sour service}

The data on the production of plates $20.0 \mathrm{~mm}$ thick for the manufacture of the X52MS, X56MS, X60MS, and X65MS grade large-diameter electric-welded pipes ordered for sour service are considered in [4]. For the reduction of the central segregation heterogeneity of the continuously cast slabs and plates, the heats contained $\mathrm{C}$ $\leq 0.07 \%$ and $\mathrm{Mn} \leq 1.00 \%$, and the mass fraction of harmful impurities was limited to the level $\mathrm{S} \leq 0.001 \%$ and $\mathrm{P} \leq 0.010 \%$. For effective sulfide globularization, calcium treatment was carried out at a $\mathrm{Ca} / \mathrm{S}$ ratio of $\sim 2$. All experimental heats were alloyed with $\mathrm{Cu}+\mathrm{Ni}+\mathrm{Cr} \leq$ $0.80 \%$, and the heats from the X60MS and X65MS grade steels additionally contained Mo. The steels were microalloyed with complex additions of $\mathrm{Ti}+\mathrm{Nb}+\mathrm{V} \leq$ $0.120 \%$. The use of the optimal chemical composition and continuous casting technology ensured the centreline segregation of slabs at a level of 1.5-2.0.

Thermomechanical processing of plates in the twostand plate mill was carried out using technology of controlled rolling followed by accelerated cooling. The start temperature of the accelerated cooling was above the phase transformation start temperature $\left(\mathrm{Ar}_{3}\right)$. The finish temperature of accelerated cooling was specified to be $520 \pm 30^{\circ} \mathrm{C}$ for the X52MS, X56MS and X60MS grade steel plates and $430 \pm 30^{\circ} \mathrm{C}$ for the $\mathrm{X} 65 \mathrm{MS}$ grade steel plates. The accelerated cooling rate was $25 \pm 5^{\circ} \mathrm{C} / \mathrm{s}$.

The plates have a fine-grained ferritic-bainitic microstructure (quasipolygonal ferrite and high-carbon bainite) with a slight central structural heterogeneity and high cleanliness from non-metallic inclusions. The mechanical properties of the plates met the specified requirements for steels. All plates exhibited a high HIC resistance and met the requirements for SSC resistance

\section{Conclusion}

Optimal concentration of segregating elements $(\mathrm{C} \leq$ $0.06 \%, \mathrm{Mn} \leq 1.00 \%$ ), and schedules of accelerated cooling after controlled rolling $\left(\mathrm{T}_{\mathrm{sc}} \geq \mathrm{Ar}_{3} ; \mathrm{T}_{\mathrm{fc}}=\right.$ $520 \pm 30^{\circ} \mathrm{C} ; \mathrm{V}_{\mathrm{c}} \geq 20^{\circ} \mathrm{C} / \mathrm{c}$ ) provide the high resistance to destruction in $\mathrm{H}_{2} \mathrm{~S}$-containing media due the formation of homogeneous ferritic-bainitic microstructure was determined. According to the research results, the technology for manufacturing of plates for largediameter pipes grades X52MS, X56MS, X60MS, and $\mathrm{X} 65 \mathrm{MS}$ ordered for sour service was developed.

\section{References}


1. Ishikawa N., Endo S. et al., Proceedings of the Microalloyed Steels for Sour Service International Seminar: CBMM, 135-154 (2014)

2. Yamada K. et al., International Conference on Technology and Applications of HSLA Steels., Philadelphia, Pensylvania, 835-842 (1983)

3. Sour gas resistant pipe steel, Niobium Information. № 18/01. CBMM/NPC, Düsseldorf, Germany (2001)

4. I. Shabalov, Y. Matrosov, A. Kholodnyi. et al., Pipeline Steels for Sour Service. Springer, Cham (2019)

5. A. Kholodnyi, Yu. Matrosov, M. Matrosov, S. Sosin, Metallurgist 60, 54-60 (2016)

6. Yu. Matrosov, A. Kholodnyi, M. Matrosov, et al., Metallurgist 59, 60-68 (2015)

7. Schwinn V., Thieme A., International Seminar «Pipe Seminar Modern Steels for Gas and Oil Transmission Pipelines, Problems and Prospects», 165-172 (2006) 